\title{
Integrating Electrical Machines and Antennas via Scalar and Vector Magnetic Potentials; an Approach for Enhancing Undergraduate EM Education
}

\author{
Seemein Shayesteh \\ Department of Electrical and \\ Computer Engineering \\ Indiana University Purdue \\ University Indianapolis \\ Indianapolis, IN \\ shayests@iupui.edu
}

\author{
Maryam Rahmani \\ Department of Electrical and \\ Computer Engineering \\ Indiana University Purdue \\ University Indianapolis \\ Indianapolis, IN \\ maryrahm@iu.edu
}

\author{
Lauren Christopher \\ Department of Electrical and \\ Computer Engineering \\ Indiana University Purdue \\ University Indianapolis \\ Indianapolis, IN \\ lauchris@iupui.edu
}

\author{
Maher Rizkalla \\ Department of Electrical and \\ Computer Engineering \\ Indiana University Purdue \\ University Indianapolis \\ Indianapolis, IN \\ mrizkall@iupui.edu
}

\begin{abstract}
This Innovative Practice Work In Progress paper presents an approach for enhancing undergraduate Electromagnetic education.
\end{abstract}

Keywords - Electromagnetic, antennas, electrical machines, undergraduate, potentials, projects, ECE

\section{INTRODUCTION}

Often times, the subject of using two potential functions in magnetism, the scalar magnetic potential function, $V_{m}$, and the vector magnetic potential function, $A$ (with zero current restriction when applying $V_{m}$ ), is a source of confusion. When using an analogous approach between the electric and magnetic potentials within an undergraduate Electromagnetic (EM) course, students usually raise the issue of dissimilarities between the electric and magnetic field equations when referring to their potential functions. Textbooks [1-3] usually do not emphasize the importance of using the vector magnetic potential, $A$, in magnetism and its role in modifying the field equations in the dynamic domain.

Three lecture periods, extended over one and a half week, were dedicated to address the analogies between the electric field $E$ and magnetic field $H$ equations, with applied EM examples. Three sets of applications were incorporated into these extended lectures. These applications are electrical machines for the scalar magnetic potential function $V_{m}$, antennas for the vector magnetic potential function $A$, and electronic devices for the (scalar) electric potential function $V$. Since the traditional EM undergraduate course serves as a pre-requisite for both Electrical Machines and Antennas courses, these extended lectures were found to be important not only for understanding the EM field equations but also for integrating them into the next upper level courses within the EE curriculum. This paper details the applied examples from both areas, for better understanding of this section of the course, and for preparing the students for the upper level courses. This work also reports the impact of using examples from electrical machines to show the importance of applying the magnetic potentials into electrical machines. This better comprehension of the magnetism section of the course was assessed by comparing students' performances within the EM course over two successive offerings of the EM undergraduate course. In one offering the course was attached with a project using COMSOL software that assisted students with better understanding of the differential EM equations within the course.

\section{COURSE OUTLINE}

\section{A. Typical EM Course within an ECE Curriculum}

A typical EM undergraduate course requires Physics (electricity and magnetism) and Differential equations as prerequisite courses and continues the study on electrostatics, magnetostatics, and Maxwell's Equations. The course also gives introduction to electromagnetic waves, transmission lines, and radiation from antennas. Some of the learning objectives cover students' ability to work with electrostatic fields and to be able to find electric and potential fields from charge distributions including the presence of dielectric materials. They should also be able to work with magnetostatic and time varying fields and find magnetic fields from current distribution, and solve wave equations to understand the wave propagation in dielectric and lossy media. The students learn how to use analogy between plane waves and transmission lines (TL) and use the transmission line analytical and graphical methods for proper and improper impedance matching in time and frequency domains. Table I summarizes the course contents for a typical Electromagnetic course.

Within the above content, the analogy between $E$ and $H$ fields may raise some issues that would need more illustration in the course. Table II shows an example of these concerns. Three lectures are added to enhance the integration of the course components (Lectures 1, 15, and 27). An attached project was also given as a bonus project.

\section{B. Elaborations on the Updated Course}

Three lectures are added to enhance the integration of the course components (Lectures 1, 15, and 27). An attached project was also given as a bonus project.

In lecture 1 (new), students, from prior electronic devices and physics classes, can see the importance of the electrostatic fields in solid state characteristics such as charge, electric fields,

Integrated Nanotechnology Development Institute (INDI). 
potentials, I-V characteristics, breakdown characteristics in PN junctions and in BJT and FET devices. It is important to solve partial differential equations to find the potentials within channels or base regions, etc. A few diagrams from a previous electronics class are used.

TABLE I. TYPICAL EM COURSE CONTENTS

\begin{tabular}{|c|c|}
\hline $\begin{array}{c}\# \text { of } \\
\text { Lectures }\end{array}$ & Topic \\
\hline 4 & $\begin{array}{l}\text { Introduction: Charge; Electric and Magnetic Fields; Units; } \\
\text { Vector Analysis; Coordinate Systems; Line, Surface and } \\
\text { Volume Integrals; Derivatives }\end{array}$ \\
\hline 4 & $\begin{array}{l}\text { Electro Statics: Static Electric Fields; Gauss' Law; } \\
\text { Coulomb's Law; Superposition; Applications of Gauss' Law } \\
\text { (Continuous Distributions of Charge); Electric Potential; } \\
\text { Conductors; Dielectrics; Displacement Field; Boundary } \\
\text { Conditions; Capacitance; Electrostatic Energy; Force; } \\
\text { Poisson's Equation; Laplace's Equation; Method of Images; } \\
\text { Electric Currents; Continuity Equation }\end{array}$ \\
\hline 4 & $\begin{array}{l}\text { Magneto Statics: Static Magnetic Fields; Lorentz Force; } \\
\text { Ampere's Law; Vector Magnetic Potential; Biot-Savart } \\
\text { Law; Magnetic Dipole; Magnetization; Magnetic Circuits \& } \\
\text { Magnetic Materials; Boundary Conditions; Inductance; } \\
\text { Magnetostatic Energy; Torque }\end{array}$ \\
\hline 6 & $\begin{array}{l}\text { Time Dependent Fields: Faraday's Law; Transformers; } \\
\text { Generators; Maxwell's Equations; Potential Functions and } \\
\text { Boundary Conditions for Time Varying Fields; Wave } \\
\text { Equation; Time Harmonic Fields. Examples from Antennas. }\end{array}$ \\
\hline 6 & $\begin{array}{l}\text { Uniform Plan Waves: Uniform Plane Wave (UPW); TEM } \\
\text { Waves; Polarization; UPW in Lossy Media; Power flow; } \\
\text { UPW Normal Incidence on Plane Boundary }\end{array}$ \\
\hline 4 & $\begin{array}{l}\text { Transmission Lines: Intro Using Parallel-Plate Transmission } \\
\text { Line; General Transmission Line Properties; Finite Length } \\
\text { Transmission Lines; Transient Behavior; Pulses }\end{array}$ \\
\hline 3 & Exams \\
\hline
\end{tabular}

TABLE II. ANALOGY BETWEEN $E$ AND $H$ FIELDS

\begin{tabular}{|l|l|l|}
\hline \multirow{2}{*}{ Analogy } & \multicolumn{2}{|c|}{ Electric Field and Magnetic Field } \\
\cline { 2 - 3 } & \multicolumn{1}{|c|}{ Electric Field $(\boldsymbol{E})$} & \multicolumn{1}{c|}{ Magnetic Field $(\boldsymbol{H})$} \\
\hline \multirow{2}{*}{$\begin{array}{l}\text { Static } \\
\text { Potentials }\end{array}$} & $\begin{array}{l}\text { Only scalar potential, } \\
E=-\nabla V \text { (unconditional) }\end{array}$ & $\begin{array}{l}V_{m}, A \\
\text { Scalar: } H=-\nabla V_{m} \\
\text { (conditionally, } J=0) \\
\text { Vector Potential, } A, \\
\text { where } B=\nabla \times A\end{array}$ \\
\hline $\begin{array}{l}\text { Laplace } \\
\text { and } \\
\begin{array}{l}\text { Poisson's } \\
\text { equations }\end{array}\end{array}$ & $\begin{array}{l}\text { Laplace within conducting } \\
\text { materials, and Poisson's } \\
\text { within dielectrics }\end{array}$ & $\begin{array}{l}V_{m} \text { satisfies Laplace, } \\
\text { while } A \text { satisfy } \\
\text { Poisson's equations }\end{array}$ \\
\hline $\begin{array}{l}\text { Dynamic } \\
\text { Fields }\end{array}$ & \multicolumn{1}{|c|}{$E=-\nabla V-\partial A / \partial t$} & $E=-\nabla V-\partial A / \partial t$ \\
\hline
\end{tabular}

Lectures 2 to 5 introduce the following topics - Charge, Electric and Magnetic Fields, Units, Vector Analysis, Coordinate Systems, Line, Surface and Volume Integrals, and Derivatives

Lectures 6 to 8 cover Electrostatics where the following topics are taught - Static Electric Fields, Gauss' Law, Coulomb's Law, Superposition, Applications of Gauss' Law (Continuous Distributions of Charge), Electric Potential, Conductors, Dielectrics, Displacement Field, Boundary Conditions, Capacitance, Electrostatic Energy, Force, Poisson's Equation, Laplace's Equation, Method of Images, Electric Currents, and
Continuity Equation. (The lectures on Coulomb and Gauss laws are combined to save one lecture from this section).

Lectures 10 to 14 cover Magnetostatics where Static Magnetic Fields, Lorentz Force, Ampere's Law, Vector Magnetic Potential, Biot-Savart Law, Magnetic Dipole, Magnetization; Magnetic Circuits \& Magnetic Materials, Boundary Conditions, Inductance, Magnetostatic Energy, and Torque are taught. Analogy between Electrostatic and Magnetostatic fields has been used as an effective approach to learn the material.

Lecture 15 (new) covers the following - applications of Magnetostatics in electrical machines and transformers, applications of the scalar magnetic potentials inside transformers cores, magnetic circuits, and the concept of DC machines.

Lectures 17 to 19 cover Time Dependent Fields where Faraday's Law, Transformers, Generators, Maxwell's Equations, Potential Functions and Boundary Conditions for Time Varying Fields, Wave Equation, and Time Harmonic Fields in spherical fields of antennas are discussed.

Lecture 20 to 26 covers Uniform Plan Waves and Transmission Lines. Uniform Plan Waves lectures teach the following topics - Uniform Plane Wave (UPW), TEM Waves, Polarization, UPW in Lossy Media, Power flow, and UPW Normal Incidence on Plane Boundary. Transmission Lines lectures cover the following topics - Intro to Using ParallelPlate Transmission Line, General Transmission Line Properties, Finite Length Transmission Lines, Transient Behavior, and Pulses.

Lecture 27 (new) covers applications of vector and scalar magnetic potentials in radiating systems as well as in electrical machines such as AC machines.

There is also a suggested project as bonus points where students use COMSOL software. Typical project examples are magnet design and solutions of wave equations over microfluidics. The project goes in parallel with the lecture materials. No lecture session was reserved for the project.

\section{The DetAILED NEW LECTURES}

\section{A. The First New Lecture}

This first new lecture shows the applications of electrostatic fields in solid state devices. This lecture builds on the electricity and magnetism physics course and uses solutions of differential equations learned from the differential equations pre-requisite math course. There are plenty of electrostatic field applications, including the solid state devices learned in the introduction of electronics class. Here are some examples:

1) In PN junction devices, the study of Electric fields, potentials, energy, current voltage DC model equations, breakdown characteristics for regular and Zener diodes.

2) This lecture also emphasizes the device capacitance and energy stored. Different types of capacitances are studied, including two parallel plate capacitors with examples in PN Junction diodes, cylindrical capacitors with examples in coaxial 
cables or transmission lines. Spherical capacitors may have usage in Supercapacitors or electrochemical capacitors for energy storage with a wide range of applications, such as hybrid electric vehicles, electronic devices, and memory backup systems. Even though this is not covered in the previous electronic classes, it may be emphasized in other elective courses with electronic fundamentals of electric and hybrid vehicle.

3) This new lecture serves as a good foundation for the BJT electrostatic devices where the voltage current characteristics are developed. Important phenomenon such as punch-through, breakdown, etc., may be understood from basic knowledge in these chapters.

4) The electrostatic field knowledge with the analytical approach with differential equations are also used for the development of the FET I-V characteristics. Understanding the operating regions of the devices and the essentials of these devices are based on the knowledge learned in these chapters. These chapters prepare students for the ECE elective courses in semiconductor devices, Analog integrated circuits, EM sensors, Micro Electro Mechanical Systems (MEMS), and Nano Electro Mechanical Systems (NEMS).

5) Another example is given for a hyperbolic PN junction by the solution of differential equation to get the charge distribution, potential function, electric field, and capacitance.

\section{B. The Second New Lecture}

This lecture shows a little bit of applications on the scalar and vector magnetic potentials. This includes examples in magnetic circuits. The concept of the reluctance and magnetomotive forces within a core structure is introduced with analogy of electrical circuits. The scalar magnetic potential, $V_{m}$, is also applied to transformers. In this application, students see that $V_{m}$ applied to regions where $J=0$ inside the core. Students are trained to calculate the required MMF (magnetomotive force) that produces certain magnetic fluxes within the core.

Examples for electrical machines also include the rotor permanent magnet synchronous machine. The calculation of the magnetic field in this machine due to the stator currents flowing in its airgap winding is briefly discussed. The vector potential, $A$, is the preferred potential to obtain the field variables, $B$ and $H$. The flux linkage of the air gap winding, and consequently the inductance, may be obtained directly from $A$.

\section{The Third New Lecture}

Generally, in antennas the radiated fields (electric and magnetic) are determined by the current distribution on the antenna as illustrated in Figure 1.

There are two approaches to determine $\vec{E}$ and $\vec{H}$. The first approach is solving Maxwell's equation directly, and the second one is using a vector potential. For the first approach, one can start from this point:

$$
\begin{gathered}
\nabla \times \vec{H}=\vec{J}+j \omega \vec{D} \\
\vec{B}=\nabla \times \vec{A}
\end{gathered}
$$

According to the vector analysis, a vector is uniquely defined with both its divergence and its curl. So we have already defined the curl of $\vec{A}$ as $\vec{B}=\nabla \times \vec{A}$. Now according to the Maxwell's equations:

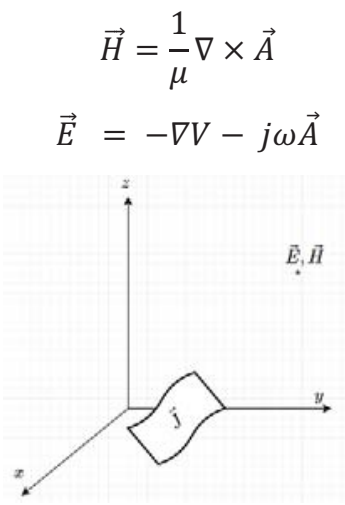

Fig. 1. E and H Fields at a desired point created by surface curent, $\vec{J}$

The wave equations for the scalar and vector potentials as developed in the course are given by:

$$
\begin{gathered}
\nabla^{2} V+\omega^{2} \mu \varepsilon=\frac{\rho}{\varepsilon} \\
\nabla^{2} \vec{A}+k^{2} \vec{A}=-\mu \vec{J}
\end{gathered}
$$

Combining Faraday's law with the energy density defined from the Poynting vector, $S$, is used to derive the antenna fields.

Different types of antennas for near field and far fields are briefly demonstrated via some simulation as shown in Figure 2.
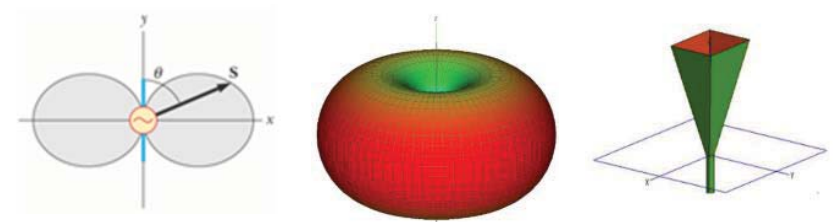

Fig. 2. Different types of antennas and the radiation patterns

The radiation power patterns as representation of the radiation properties at a given distance from the antenna can give amplitude $E$, and $H$ fields that could be plotted in $\mathrm{dB}$ as shown in Figure 3.
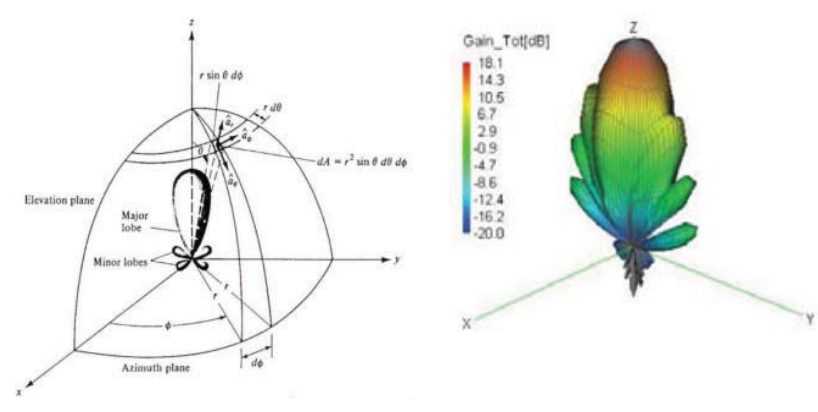

Fig. 3. Antenna radiation power patterns [4] 
Students have the option to simulate any of these antennas on HFSS software as an associated project.

\section{The AtTACHEd Project}

This covers the use of Matlab to determine:

- $\mathrm{E}$ and $\mathrm{H}$ distribution for charge and current distribution

- 3D permanent magnets (via COMSOL)

- Voltage Induced in a Coil by a Moving Magnet (via COMSOL)

- $\quad$ RF Diploe antenna (via COMSOL)

- Dipole antenna via ANSYS software

- The Yagi-Uda antenna (use of HFSS)

The project was given for bonus credit with up to $3 \%$ of the course.

The class size is around 55 students. Even though the project was given for bonus, majority of students have performed the project. Students see practical applications of the course materials that enhance their interests and encourage them to learn more about how the EM models were developed. The students worked in groups. They were given the option to choose from among several projects that may be performed with CONSOL or others. Students work under the supervision of the class TA, and sometimes they receive assistance from graduate students who are conducting research in this area. The project presentation has been optional, however, it is in the future plan to make it required.

\section{ASSESSMENT RESULTS AND DISCUSSIONS}

The new modifications were assessed via the average course grades taken over three terms: fall 2015 with no modifications, fall 2016 with new lectures and the attached project, and fall 2017 with new lectures but no project. The results are summarized in Table III which shows, based on the data taken, the impact of these modifications on students' performances.

TABLE III. SUMmary OF ASSESSEd Data OVER ThreE TERMS

\begin{tabular}{|l|c|c|c|}
\hline \multicolumn{1}{|c|}{ Assessed Data } & Group \#1 & Group \#2 & Group \#3 \\
\hline Term & Fall 2015 & Fall 2016 & Fall 2017 \\
\hline Number of Students & 58 & 51 & 48 \\
\hline Course Structure & $\begin{array}{l}\text { No new } \\
\text { lectures, } \\
\text { no project }\end{array}$ & $\begin{array}{l}\text { New } \\
\text { lectures and } \\
\text { project }\end{array}$ & $\begin{array}{l}\text { New } \\
\text { lectures, } \\
\text { no project }\end{array}$ \\
\hline $\begin{array}{l}\text { Electrostatic Fields Average } \\
\text { Score (out of 30) }\end{array}$ & 18.5 & 20 & 17.5 \\
\hline $\begin{array}{l}\text { Magnetostatics Average Score } \\
\text { (out of 30) }\end{array}$ & 16 & 20.5 & 20 \\
\hline EM Average Score (out of 30) & 19.5 & 21 & 21 \\
\hline $\begin{array}{l}\text { Total Average Score (out of } \\
\text { 90) }\end{array}$ & 54 & 61.5 & 58.5 \\
\hline Total Average Score (\%) & 60 & 68 & 65 \\
\hline
\end{tabular}

It is clear that group 2 (with new lectures and projects) scored $8 \%$ higher than group 1 . Apparently, the attached project has impacted the students' understanding. Group 3 average score improved by $5 \%$ compared to group 1 . The new lectures may have impacted this improvement.

The above assessment data does not incorporate the students' quality that may vary from one semester to another. Students' feedback, however, recommended more of software projects to enhance their understanding of the various sections of the course. COMSOL and HFSS helped some of the students to pursue further research in EM.

More data with project based offerings will be collected for future assessment of the course.

\section{CONCLUSION}

The impact of the associated projects using software simulation packages such as COMSOL, HFSS, and/or ADS/EMPro will be further assessed for better understanding with some course sections that have low students' scores. Projects of high frequency modeling with semiconductor devices may also be included.

The current course considers the project as bonus credit, and this will change to be an integral part of the course grading system to insure hands on experience be delivered to all students. The future offering may incorporate ideas from conversion of electrical energy to physical motion via permanent Magnets, motors, etc., and more examples from microwave applications such as microwave ovens will be included. Within the extra lectures proposed here, a short presentation on some research topics using the software systems listed above will be included in order to attract students' interests into future EM research.

\section{ACKNOWLEDGMENT}

The authors appreciate the help of the Integrated Nanotechnology Development Institute (INDI) for their support in using COMSOL and ADS/EmPro software.

\section{REFERENCES}

[1] Kenneth Demarest, Engineering Electromagnetics, Prentice- Hall,1998

[2] William Hayt and John Buck, Engineering Electromagnetics, McGraw Hill, 2010

[3] Fawwaz T. Ulaby, Electromagnetic for Engineers, Prentice Hall, 2005

[4] Balanis, Constantine A. "Antenna theory analysis and design, 1997." International Journal of Aerospace Engineering Hindawi Publishing Corporation http://www. hindawi. com 2010. 